PAPER

\title{
Clinical and serological study of myasthenia gravis in HuBei Province, China
}

\author{
Xiaofan Zhang, Mingshan Yang, Jinzhi Xu, Min Zhang, Bethan Lang, \\ Wei Wang, Angela Vincent

\section{Correspondence to:}

Professor A Vincent,

Neurosciences Group

Weatherall Institute of

Molecular Medicine, John

Radcliffe Hospital, Oxford

OX3 9DS, UK; angela.

vincent@imm.ox.ac.uk

Co-corresponding author:

Professor Wei Wang,

Department of Neurology,

Tongji Hospital, Huazhong

University of Science and

Technology (HUST), WuHan,

China; wwang@tjh.tjmu.

edu.cn

Received 22 June 2006

Revised 13 October 2006

Accepted 23 October 2006

Published Online First

6 November 2006

J Neurol Neurosurg Psychiatry 2007;78:386-390. doi: 10.1136/innp.2006.100545

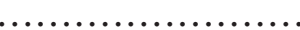

Background: Ocular and childhood myasthenia gravis (MG) cases seem relatively more common in Oriental than in Caucasian populations, but there have been no comprehensive serological studies on patients from mainland China.

Methods: 391 unselected patients with MG attending Tongii Hospital in WuHan (the largest hospital in the province of HuBei, China) were studied during a 15-month period; most had already received treatment for their condition.

Results: The male to female ratio was 0.8 . $50 \%$ of the patients were children ( $<15$ years), and age at onset showed a single peak at between 5 and 10 years of age. $64 \%$ of the children and $66 \%$ of the adults were positive for acetylcholine receptor (AChR) antibodies but the antibody titres were lower than in similar Caucasian studies, although this was partly due to the high incidence of ocular MG. Of the 43 patients with generalised MG without AChR antibodies, only 1 had muscle-specific kinase antibodies (2.5\%) and 2 had voltage-gated calcium channel antibodies indicating probable Lambert-Eaton myasthenic syndrome. $75 \%$ of the children, compared with only $28 \%$ of the adults, had ocular MG. Thymoma was evident by MRI in $1.5 \%$ of children and in $20 \%$ of adults. Despite most patients having received prednisone, very few had obtained full clinical remission.

Conclusion: This study emphasises the frequency of early childhood onset with ocular symptoms and shows that many of these patients have AChR antibodies. By contrast, patients presenting in later age seem to be very uncommon in comparison with recent studies in Caucasian populations.
M yasthenia gravis (MG) is the most common primary disorder of neuromuscular transmission. Acetylcholine receptor (AChR) antibodies are present in sera from $80 \%$ to $90 \%$ of patients with generalised $M G$, about $50 \%$ from those with pure ocular MG and infrequently in healthy people. ${ }^{1}$ The remaining $10-20 \%$ of generalised patients with MG are AChR antibody negative (seronegative MG, SNMG). IgG autoantibodies to the muscle-specific kinase (MuSK) were first identified in 70\% of patients with generalised SNMG. ${ }^{2}$ However, subsequent reports have found a variable prevalence in different countries, with a low proportion of MuSK antibody-positive MG in one study from Taiwan ${ }^{3}$ (reviewed in Vincent and Liete ${ }^{4}$ ).

A number of studies indicate that MG in Oriental populations may be clinically different from that in Caucasians. ${ }^{5-8}$ In particular, paediatric cases are frequent in China, Taiwan and Japan and purely ocular MG is relatively common in children. Since there are also differences in human leucocyte antigen associations between Japanese, Chinese and Caucasian populations, ${ }^{9-12}$ these observations may provide clues to the immunopathogenesis of MG.

However, there have been no comprehensive studies of AChR and MuSK antibodies in patients from mainland China. Here, we studied the histories and serology of 391 Chinese patients attending a major hospital in WuHan, China.

\section{PATIENTS AND METHODS}

During a 15-month period (September 2004-December 2005), all 600 patients with a diagnosis of MG presenting to the Tongji Hospital, WuHan, China, were invited to participate, and 391 consented to give serum for antibody studies. A total of 324 patients had been monitored regularly in our hospital over periods from $<1$ to 27 years, and 67 were attending for the first time. The diagnosis of MG was made on the basis of the clinical history of fatiguable weakness, neostigmine test and the response to acetylcholinesesterase therapy. Electromyography was only performed in 2 children and 10 adults. The severity was described according to the Myasthenia Gravis Foundation of America (MGFA) classification ${ }^{13}$ (for quantitative purposes, grades I-V were changed to 1-5). Thymic pathology was deduced from MRI scans, but thymectomy was only performed in 44 patients.

AChR antibodies and MuSK antibodies were measured using radioimmunoprecipitation assays (RSR, Cardiff, UK). AChR antibody values $>2.5 \mathrm{nM}$ were re-assayed with $\mathrm{l} \mu \mathrm{l}$ of serum to provide a more accurate titre. Voltage-gated calcium channel (VGCC) antibodies were measured as described previously. ${ }^{14}$ Serum from 20 healthy individuals and 80 patients with other diseases were used as controls.

\section{RESULTS}

Demographic and serological features of all patients with MG

The most striking feature was the large number of children with MG, many aged $<5$ years at the first recognition of symptoms (fig 1A). In all, 50\% (197/391) of the patients presented as children aged <14 years and 50\% (194/391) as adolescents or adults; only 6 patients were aged $>60$ years (fig 1A). The male-to-female ratio was 0.8 , with no clear difference in sex distribution at any age (fig 1A).

Abbreviations: $A C h R$, acetylcholine receptor; $M G$, myasthenia gravis; MGFA, Myasthenia Gravis Foundation of America; MuSK, muscle-specific kinase; SNMG, seronegative myasthenia gravis; VGCC, voltage-gated calcium channel 


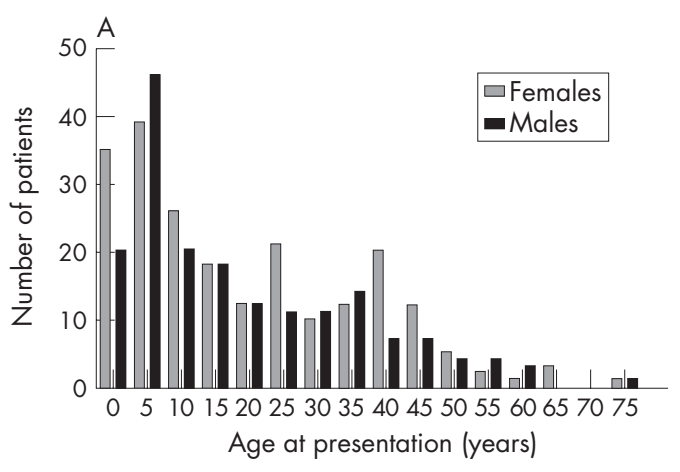

AChR antibodies were tested in all 391 sera, although many of the patients had had MG for many years (range 1-45 years) at the time of sampling. After subtraction of the mean of three healthy Chinese sera, the Chinese control values ranged from -0.1 to $0.35 \mathrm{nM}$. A conservative value of $0.5 \mathrm{nM}$ was used to distinguish AChR antibody-positive from AChR antibodynegative patients (as used in the Oxford laboratory). AChR antibodies were positive in only 65\% (254/391) of the Chinese patients with MG (fig 1B). Overall, the AChR antibody levels were lower than they are in typical AChR antibody-positive Caucasian patients with MG (data not shown), and there was no clear demarcation between positive and negative values within the patient population.

Three cases of familial MG were present among the cohort (approximately 1\%): a father and son, a father and daughter, and a mother and son. In all cases, both individuals were positive for AChR antibodies. A mother with AChR antibodies and her newborn baby were both positive. Seven healthy siblings of patients with MG were all negative for AChR antibodies.

Only 1 of the $43(2.5 \%)$ AChR antibodies-negative patients with generalised MG was positive for MuSK antibody, and none of the patients with ocular MG was positive for MuSK antibodies (see below). MuSK antibodies were also not detected in 65 patients with AChR antibodies. Two patients were positive for VGCC antibodies. All three patients with MuSK or VGCC antibodies were excluded from further analysis, but their histories are summarised below.

\section{Clinical features}

The presenting symptoms were ocular motor disturbance in $73 \%$, oropharyngeal weakness in $18 \%$ and limb weakness in $9 \%$. Only $16 \%(62 / 388)$ of the patients had progressive weakness that involved oropharyngeal and limb muscles. We used the patients' records to assign MGFA scores at onset, maximum weakness and at the last clinic visit. At the time of serum sampling, positivity for AChR antibody was $65 \%$ in all patients, with $59 \%$ in purely ocular $M G$, and $85 \%$ in patients with grades $\geqslant 2$ a (fig 2 ).

The duration of MG at the time of study ranged widely, from $<1$ to 45 years (median 3 years). One-way analysis of variance showed some influence of disease duration on MGFA grade ( $p=0.02$ for adults and $p=0.04$ for children), but post-testing showed that only the grades in adult patients with $>10$ years of disease duration were significantly different from the presenting MGFA grade $(\mathrm{p}<0.05)$. We therefore ignored duration of disease in further analyses.

Although most patients presented with ocular MG (MGFA grade 1), many adult patients with AChR antibodies had generalised disease at onset (fig 2) or progressed to generalised
Figure 1 (A) Frequency histograms of age at presentation for male and female Chinese patients with myasthenia gravis (MG). (B) Acetylcholine receptor (AChR) antibody levels in 100 Chinese controls and in 391 patients with MG. The line is drawn at $0.5 \mathrm{nM}$, which is the cut-off used in the Oxford laboratory where the assays were performed.

\section{Thymus pathology}

All the patients had MRI scans to look for thymic pathology; the results were classified as normal for age, thymitis or thymoma. Most children had a normal thymus but a proportion had thymitis evident on scans (fig 3); only 15 patients with thymitis (aged 3-52 years) underwent a thymectomy. Overall, 42 patients $(11 \%)$ had thymoma detected on scans; 3 of these were AChR antibody-positive children. In all, 39/191 (20\%) adult patients had thymomas, 4 of these were negative for AChR antibodies. In the 28 patients with thymoma who underwent thymectomy, the pathology was confirmed by histology. None have had a thymoma recurrence during a follow-up period of a median of 3 years.

\section{Response to treatments}

Most patients were given acetylecholinesterase inhibitors (pyridostigmine bromide) at doses between 120 and $180 \mathrm{mg} /$ day. Most patients also received prednisone at doses starting at $30 \mathrm{mg} /$ day, reducing to $10-15 \mathrm{mg} /$ day within a few weeks if there was improvement. These doses were then maintained for 6 months to 1 year, after which they were reduced to $5 \mathrm{mg}$ /day. Patients who did not show or report clinical benefit received azathioprine and intravenous immunoglobulin, and occasionally plasma exchange. Most patients improved to some extent with prednisone, but it was usually difficult to reduce this dose beyond $5 \mathrm{mg} /$ day.

Despite the prednisone treatments, many of the adults with AChR antibodies still had generalised disease and no patients were in complete clinical remission (MGFA grade 0 ) at the time of the study. Using the post-interventional scoring system, only one patient was known to have died, 11 were worse, 151 were unchanged and 225 had improved. The final MGFA grades were 


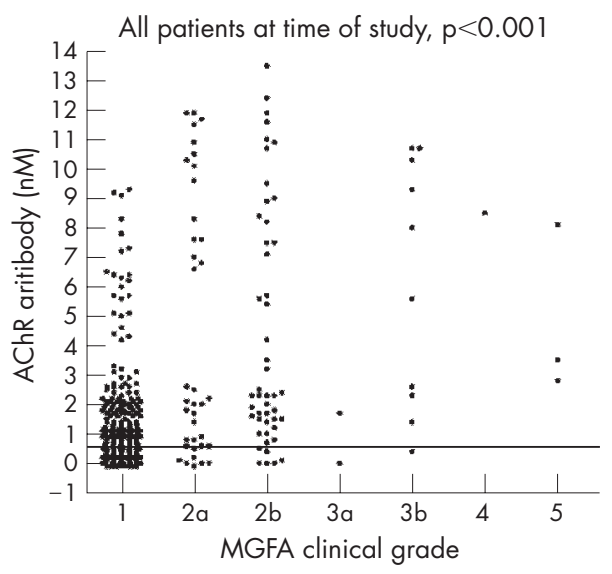

Figure 2 Acetylcholine receptor (AChR) antibody levels in Chinese patients plotted against Myasthenia Gravis Foundation of America (MGFA) clinical grades at the time of sampling (given as $1-5$, corresponding to $1-$ V). Most of the AChR antibody-negative samples were from patients with MGFA grade 1. Some of these patients had had higher grades at maximum severity (table 1).

significantly lower than the grades at maximum severity for both children and adults, but the greatest change was in adults who undervent thymectomy (fig 4).

\section{Patients with MuSK or VGCC antibodies}

Only 1 of 44 generalised patients with SNMG $(2.5 \%)$ was positive for MuSK antibody. This patient was a 45-year-old woman who developed transient ptosis and diplopia followed by progressive dysphagia, dysarthria and limb weakness. No thymic abnormalities were identified on MRI scan. The patient did not respond well to pyridostigmine. She developed severe dyspnoea requiring ventilatory support. Because of progressive dyspnoea, the patient underwent three sessions of immunoadsorption, with a dramatic response. During the 1-year follow-up period, she was much improved and led a normal life when she was maintained on pyridostigmine $180 \mathrm{mg} /$ day and prednisone $15 \mathrm{mg} /$ day.

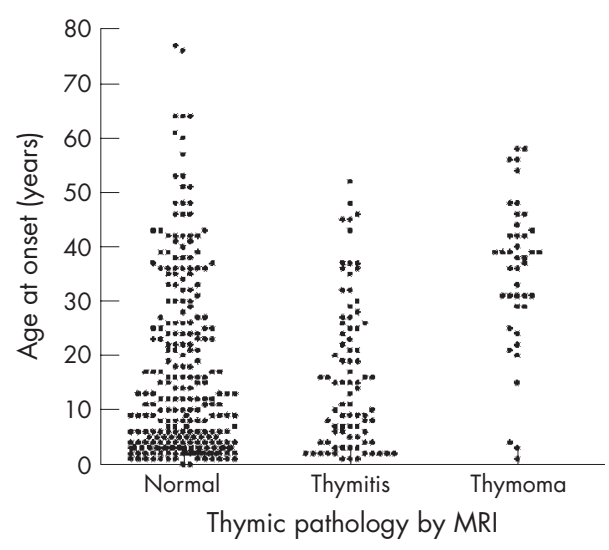

Figure 3 Age at onset plotted against thymic pathology. MRI was used to define the thymic pathology since many patients were not thymectomised.

The patients with VGCC antibodies were both men, aged 40 and 49 years at onset. Both had relatively mild generalised disease for 7 years and 1 year, with no evidence of lung cancer on CT scan of the thoraces. Only one received prednisone, and both are unchanged at present.

\section{Factors that might influence the clinical expression of MG in China}

A total of 21 of the children had hyperthyroidism (diagnosed on the basis of FT3, FT4 and thyroid-stimulating hormone levels), 16 of whom were AChR antibody positive (76\%). In addition, 33 of the adults had hyperthyroidism, of whom 50\% were AChR antibody positive at the time of study. There were no other relevant disease associations in the patients. To look at the demographics of MG in China, we comapred the total MG cases with the demographics of the total population in HuBei Province (table 2). The prevalence of MG in children (14/million) was higher than that in adults (4.5/ million), although this might partly reflect the self-referral patterns to different hospitals in the province.

The patients described above include 67 newly referred patients who had not received treatment before, and we added a further 40 patients seen in 2006. In HuBei Province, 40\% (24

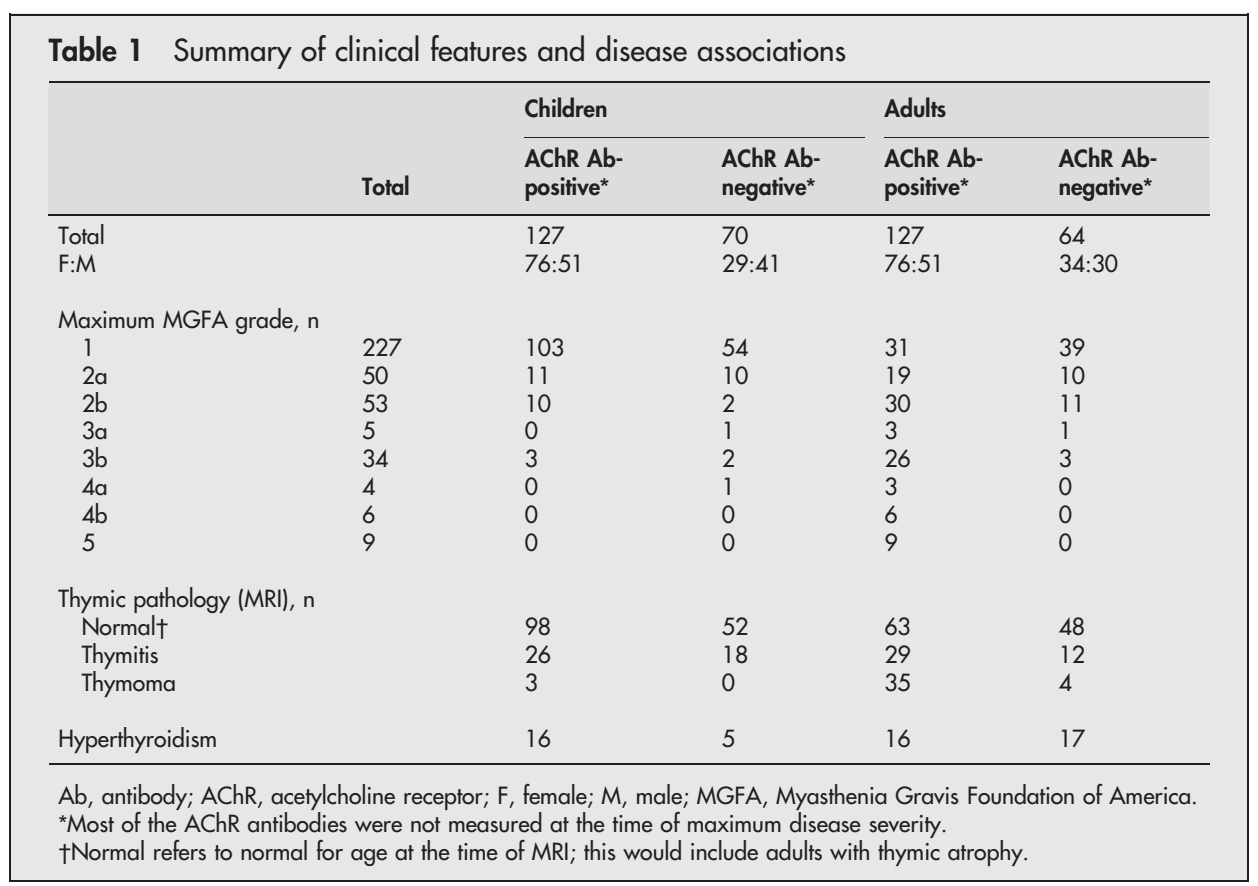



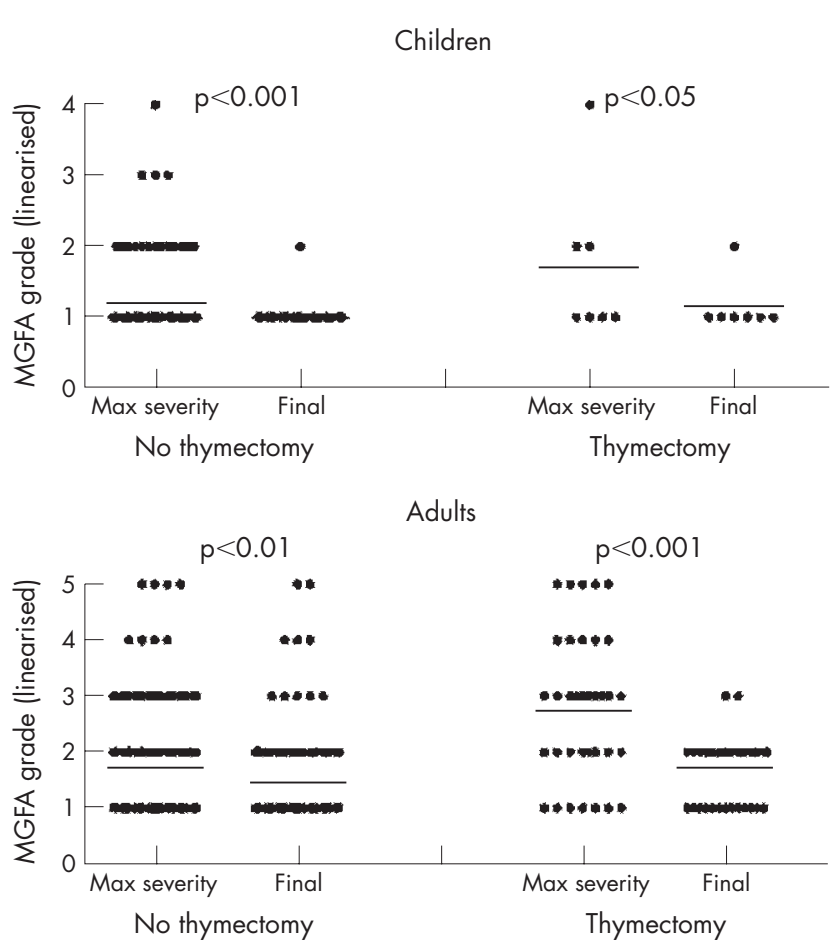

Figure 4 The Myasthenia Gravis Foundation of America (MGFA) grades (given as 1-5, corresponding to I-V) are shown at maximum (max) severity and final follow-up in patients without or with thymectomy.

million) of the 60 million population lives in rural villages (table 3). We found that among the 107 newly presenting patients at the Tongji Hospital, 54\% came from rural communities and $46 \%$ from urban communities, both children and adults. Striking, however, was the time between onset of symptoms and the first visit to the clinic in many cases, with a suggestion of longer time intervals in females. The longest duration from the onset to the first clinic was in a rural woman who had symptoms for 47 years.

\section{DISCUSSION}

MG occurs worldwide, ${ }^{15}$ but differences in the clinical expression of MG have been observed, principally between Caucasian and Oriental patients. This was the first large clinical/serological study of unselected patients with MG attending a clinic in mainland China. The most striking result is the high proportion of childhood cases, mostly with purely ocular MG. In contrast with most Caucasian studies, there were few patients aged $>40$ years presenting to the clinic and no marked gender bias, which differs from that in Caucasians. ${ }^{15-18}$ AChR antibodies were found in $>60 \%$ of the patients with ocular MG, both children and adults, and in $85 \%$ of those with generalised disease at the time of sampling. Thus, we confirm that MG in mainland China differs from that in Caucasian populations by the high incidence of childhood ocular MG, and show that a high proportion of these childhood cases are AChR antibody positive.

Despite the high positivity in ocular MG cases, overall, 35\% of the patients were AChR antibody negative, which is higher than the previous results $(13 \%)$ of the Chinese patients in Taiwan. ${ }^{5}$ However, that study only performed AChR antibody titres on 67 untreated patients, rather than the full cohort of 388 patients with MG studied here, and it was not clear whether they were adult or childhood cases. Moreover, many of our patients had a long disease duration and were not at maximal severity at the time of serum sampling. Alternatively,
Table 2 Number of patients with myasthenia gravis related to the population of HuBei Province

\begin{tabular}{llll}
\hline & $\begin{array}{l}\text { Total patients } \\
\text { with MG }\end{array}$ & $\begin{array}{l}\text { HuBei Province* } \\
\text { (millions) }\end{array}$ & $\begin{array}{l}\text { Patients with } \\
\text { MG (per million) }\end{array}$ \\
\hline Total population & 388 & 60 & 6.47 \\
Male & 173 & 31 & 5.58 \\
Female & 15 & 28 & 7.68 \\
& & & \\
Age (years) & 197 & 14 & 14.07 \\
0-14 & 189 & 42 & 4.50 \\
15-64 & 2 & 3.7 & 0.54 \\
965 & & &
\end{tabular}

MG, myasthenia gravis.

*These data are based on the website of the National Bureau of Statistics of China.

one could argue that the seronegative cases may not have had MG, since electromyography is not carried out routinely at our hospital, and was performed in only 12 patients. Moreover, the neostigmine test is unlikely to have the sensitivity or specificity profile of the AChR antibody. ${ }^{19}$ The high incidence of ocular MG cases will confound this problem as they are known to have lower positivity on all tests. Nevertheless, here, only three cases were found to have other neuromuscular junction disorders on serological testing of the patients with generalised AChR antibody-negative $\mathrm{MG}$, and the proportion of AChR antibody positivity among the ocular cases is similar or higher than that found in Western studies ${ }^{20}$. Therefore, most of the AChR antibody-negative cases are likely to have had myasthenia. On the other hand, it is possible that some of those cases designated as ocular MG had mild generalised weakness that was unrecognised; this possibility requires further study.

We detected MuSK antibodies in only 1 of $44(2.5 \%)$ of our Chinese patients with generalised SNMG, which is similar to the result $(4 \%)$ reported previously in Taiwan. ${ }^{5}$ This frequency is much lower than the $40-70 \%$ frequency reported in most Caucasian studies, ${ }^{21-24}$ although a recent study from Norway found no MuSK antibody-positive patients. ${ }^{25}$ This regional variation, therefore, applies to both Western and Eastern populations $^{4}$ (Avincent, unpublished results, 2006). Like many previously reported Caucasian MuSK antibody-positive patients, ${ }^{21-24}$ our Chinese MuSK antibody-positive patient was an adult women with marked bulbar weakness. We also found two patients with VGCC antibodies which were diagnostic for Lambert-Eaton myasthenic syndrome. This syndrome has not been widely considered in the differential diagnosis of neuromuscular weakness in China, and had not been diagnosed clinically. Fortunately, neither patient has developed lung cancer.

Table 3 Demographics of 107 patients at first presentation

\begin{tabular}{|c|c|c|}
\hline & Rural & Urban \\
\hline $\begin{array}{l}\text { Total population in HuBei Province } \\
\text { (millions) }\end{array}$ & 24 & 36 \\
\hline Females with $M G, n$ & 27 & 27 \\
\hline Males with $M G, n$ & 30 & 23 \\
\hline \multicolumn{3}{|l|}{ Age at onset } \\
\hline $0-14$ years, $n$ & 25 & 20 \\
\hline$>14$ years, $n$ & 32 & 30 \\
\hline \multicolumn{3}{|l|}{ Duration from onset to first clinic } \\
\hline$<1$ year, $n$ & 33 (F:13; M:20) & $30(\mathrm{~F}: 13 ; \mathrm{M}: 17)$ \\
\hline $1-3$ years, $n$ & $17(\mathrm{~F}: 9 ; \mathrm{M}: 8)$ & 15 (F:11;M:4) \\
\hline$>3$ years, $n$ & 7 (F:5; M2) & 5 (F:3; M:2) \\
\hline Hyperthyroidism, n & 9 & 6 \\
\hline
\end{tabular}


The thymic pathology was assessed by MRI, and for economic reasons thymectomy was only performed in a proportion of patients, even those with thymitis or thymoma. In all those who undervent thymectomy, the pathology was confirmed by histology. However, since no cases with "normal" pathology on MRI were treated surgically, we cannot assess the sensitivity of the MRI. Although, unusually, four of the patients with a history of MRI-diagnosed thymomas (three confirmed by histology) were negative for AChR antibodies; sera from the time of diagnosis were not available.

Apart from the study from Taiwan, ${ }^{5}$ the closest is that of 470 Japanese patients. $^{8}$ Previous reports have indicated a high frequency of human leucocyte antigen class II allele-DR9 in both Chinese and Japanese patients, ${ }^{9-12}$ compared with DR $3^{18}$ in Caucasians, so one might expect the clinical expression to be similar. The Japanese patients were recruited from 19 Japanese tertiary medical centres and included both childhood and adult cases, but the median age at onset was in adulthood, differing from that shown here. In addition, they found fewer patients with purely ocular MG and most of the Japanese patients achieved remission, with $30 \%$ of the patients achieving complete remission (MGFA grade 0 ), whereas this was not the case in our patients. One of the reasons could be that more of their patients underwent thymectomy (68\% in Japan, $11.25 \%$ in China), since we found here that there was greater improvement in patients who had undergone thymectomy than those who had not (fig 4), although these results are confounded by the use of prednisone in most patients. Another reason for the difference in clinical response may be that the Chinese patients were younger than the Japanese. Childhood MG is so rare in Caucasian societies that the optimal treatment for childhood MG is not well studied. In China, many children receive prednisone, but the lack of clear clinical remission in our patients suggests that this treatment is not optimal. Moreover, it could stunt growth, which must be considered when starting treatment.

It is difficult to comment on the overall prevalence of MG in China from these and previous studies. ${ }^{726}$ Previous studies found a high prevalence of childhood and ocular MG cases (around 1:16 000) as we did, but did not use standard assays for antibody detection. However, despite the fact that our hospital is one of the five largest hospitals in WuHan where patients can attend a neurology clinic, the numbers of patients is likely to be an underestimate. The relatively high proportion of males compared with females at all ages, and the long duration of symptoms in a high proportion of the patients at first clinic, may reflect the fact that attendance at the neurology clinic depends on self-referral and financial situation rather than necessarily on clinical need.

Finally, these data not only indicate a need for more studies of MG and its treatment in Chinese children, but also raise interesting questions on the aetiology of this disease. The relatively high prevalence of MG in children compares strikingly with the situation in Caucasian populations ${ }^{16}$ and this suggests that the disease might follow a common childhood infection or other environmental factors. However, there was no evidence for MG segregating with rural or urban communities, making this less likely. Genetic factors, as mentioned above, may contribute to the differences in aetiology, clinical expression and susceptibility between Caucasian and Oriental races. It will, therefore, be interesting to compare the relative numbers of Chinese childhood and adult cases in other parts of the world where Chinese people reside.

\section{ACKNOWLEDGEMENTS}

We thank all the patients for participating in this study, and RSR Ltd Cardiff UK for some of the diagnostic assay kits and for arranging their transport.

\section{Authors' affiliations}

Xiaofan Zhang, Mingshan Yang, Jinzhi Xu, Min Zhang, Wei Wang, Department of Neurology, Tongji Hospital, Huazhong University of Science and Technology, WuHan, China

Xiaofan Zhang, Bethan Lang, Angela Vincent, Neurosciences Group, Weatherall Institute of Molecular Medicine, John Radcliffe Hospital, Oxford,UK

Competing interests: The Department of Clinical Neurology in Oxford receives payments and royalties from AChR and MUSK antibody tests.

\section{REFERENCES}

1 Lindstrom JM, Seybold ME, Lennon VA, et al. Antibody to acetylcholine receptor in myasthenia gravis: prevalence, clinical correlates, and diagnostic value. 1975 classical article. Neurology 1998;51:933-9.

2 Hoch W, McConville J, Helms S, et al. Auto-antibodies to the receptor tyrosine kinase MuSK in patients with myasthenia gravis without acetycholine receptor antibodies. Nat Med 2001;7:365-8

3 Yeh J-H, Chen W-H, Chiu H-C, et al. Low frequency of MuSK antibody in generalized seronegative myasthenia gravis among Chinese. Neurology 2004;62:2131.

4 Vincent A, Leite MI. Neuromuscular junction autoimmune disease: muscle specific kinase antibodies and treatments for myasthenia gravis. Curr Opin Neurol 2005; 18:519-25.

5 Chiu H-C, Vincent A, Newsom-Davis J, et al. Myasthenia gravis: population differences in disease expression and acetylcholine receptor antibody titers between Chinese and Caucasians. Neurology 1987;37:1854-7.

6 Fukuyama Y, Hirayama Y, Osawa M. Epidemiological and clinical features of childhood myasthenia gravis in Japan. In: Satyoshi E, eds. Myasthenia gravis pathogenesis and treatment. Tokyo: University of Tokyo Press, 1981:19-27.

7 Xu J, Yang M, Li B, et al. Myasthenia gravis: clinical study in 2385 patients. Chin J Neurol 1999;32:347-50.

8 Kawaguchi N, Kuwabara S, Nemoto $Y$, et al. Treatment and outcome of myasthenia gravis: retrospective multi-center analysis of 470 Japanese patients, 1999-2000. J Neurol Sci 2004;224:43-7.

9 Shinomiya N, Nomura Y, Segawa M. A variant of childrenhood-onset myasthenia gravis: HLA typing and clinical characteristics in Japan. Clin Immunol 2004; 110:154-8.

10 Hawkins BR, Chan-Lui WY, Choi EKK, et al. Strong association of HLA BW46 with juvenile onset myasthenia gravis in Hong Kong Chinese. J Neurosurg Psychiatr 1984:47:555-7.

11 Chen WH, Chiu HC, Hseih RP. Association of HLA-Bw46DR9 combination with juvenile myasthenia gravis in Chinese. I Neurol Neurosurg Psychiatr 1993;56:382-5.

12 Matsuki K, Juji T, Tokunaga K, et al. HLA antigens in Japanese patients with myasthenia gravis. J Clin Invest 1990:86:392-9.

13 Jaretzki A III, Barohn RJ, Ernstoff RM, et al. Myasthenia gravis: recommendations for clinical research standards. Task force of the Medical Scientific Advisory Board of the Myasthenia Gravis Foundation of America [review]. Neurology 2000;55:16-23.

14 Motomura M, Lang B, Johnston I, et al. Incidence of serum anti-P/O-type and anti-N-type calcium channel autoantibodies in the Lambert-Eaton myasthenic syndrome. J Neurol Sci 1997;147:35-42.

15 Kurtzke JF, Kurland LT. In:, Baker AB, Baker LH, eds. Epidemiology of neurologic disease. Hagerstown, MD: Harper \& Row, 1977:3.

16 Vincent A, Clover L, Buckley C, et al. Evidence of underdiagnosis of myasthenia gravis in older people. J Neurol Neurosurg Psychiatry 2003;74:1105-8.

17 Mantegazza R, Baggi F, Antozzi C, et al. Myasthenia gravis (MG): epidemiological data and prognostic factors. Ann NY Acad Sci 2003;998:413-23.

18 Compston DAS, Vincent A, Newsom-Davis J, et al. Clinical, pathological, HLA antigen and immunological evidence for disease heterogeneity in myasthenia gravis. Brain 1980;103:579-601.

19 Vincent A, Bowen J, Newsom-Davis J, et al. Seronegative generalised myasthenia gravis: clinical features, antibodies, and their targets. Lancet Neurol 2003;2:99-106.

20 Kupersmith MJ, Latkany R, Homel P. Development of generalized disease at 2 years in patients with ocular myasthenia gravis. Arch Neurol 2003;60:243-8.

21 Sanders DB, El-Salem K, Massey JM, et al. Clinical aspects of MuSK antibody positive seronegative MG. Neurology 2003;60:1978-80.

22 Scuderi F, Marion M, Colonna L, et al. Anti-p1 10 autoantibodies identify a subtype of "seronegative" myasthenia gravis with prominent oculobulbar involvement. Lab Invest 2002;82:1139-46.

23 Evoli A, Tonali PA, Padua L, et al. Clinical correlates with anti-MuSk antibodies in generalized seronegative myasthenia gravis. Brain 2003;126:2304-11.

24 McConville J, Farrugia ME, Beeson D, et al. Detection and characterization of MuSK antibodies in seronegative myasthenia gravis. Ann Neurol 2004;55:580-4.

25 Romi F, Aarli JA, Gilhus NE. Seronegative myasthenia gravis: disease severity and prognosis. Eur J Neurol 2005;12:413-18.

26 Chung B, Wong V, Ip P. Prevalence of neuromuscular diseases in Chinese children: a study in southern China. J Child Neurol 2003;18:217-19. 